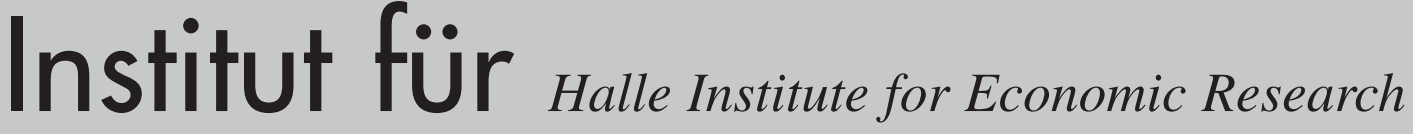 Wirtschaffsforschung Halle
}

\section{Grenzen des Wettbewerbs} im Gesundheitswesen

\author{
Ingmar Kumpmann
}

Januar 2008

Nr. 1

\section{IWH-Diskussionspapiere IWH-Discussion Papers}




\title{
Grenzen des Wettbewerbs im Gesundheitswesen
}

\author{
Ingmar Kumpmann
}

Januar 2008

Nr. 1 
Autor: Dr. Ingmar Kumpmann

Abteilung Makroökonomik

Email: Ingmar.Kumpmann@iwh-halle.de

Tel.: (0345) 77 53-705

The responsibility for discussion papers lies solely with the individual authors. The views expressed herein do not necessarily represent those of the IWH. The papers represent preliminary work and are circulated to encourage discussion with the author. Citation of the discussion papers should account for their provisional character; a revised version may be available directly from the author.

Comments and suggestions on the methods and results presented are welcome.

IWH-Discussion Papers are indexed in RePEc-Econpapers and in ECONIS.

Herausgeber:

INSTITUT FÜR WIRTSCHAFTSFORSCHUNG HALLE - IWH

Prof. Dr. Ulrich Blum (Präsident), Dr. Hubert Gabrisch (Forschungsdirektor)

Das IWH ist Mitglied der Leibniz-Gemeinschaft

Hausanschrift: Kleine Märkerstraße 8, 06108 Halle (Saale)

Postanschrift: Postfach 1103 61, 06017 Halle (Saale)

Telefon:

(0345) 77 53-60

Telefax:

(0345) 77 53-8 20

Internetadresse: $\quad$ http://www.iwh-halle.de 


\title{
Grenzen des Wettbewerbs im Gesundheitswesen*
}

\begin{abstract}
Many health economists demand more competition in the health care system. They focus on the competition between sickness funds for insured and the competition between health care providers for contracts with sickness funds. But they neglect the competition between health care providers for patients which is crucial for medical quality. This third field of competition is in conflict with the two former fields. The empirical evidence concerning the effects of competition on cost and quality is also ambiguous. Thus the mere claim for "more competition" does not do justice to the high complexity of the health care system.
\end{abstract}

Key words: health care system, competition, sickness funds, free choice of medical practitioner

JEL Codes: I11, I18, D61

\section{Zusammenfassung}

Viele Gesundheitsökonomen fordern mehr Wettbewerb im Gesundheitswesen. Damit ist ein stärkerer Wettbewerb zwischen Krankenkassen um Versicherte und zwischen Leistungserbringern um Verträge mit Kassen gemeint. Vernachlässigt wird dabei jedoch der für die medizinische Qualität wichtige Wettbewerb der Leistungserbringer um Patienten. Dieser steht mit den beiden zuerst genannten Wettbewerbsfeldern im Konflikt. Auch die vorhandene empirische Evidenz ist uneindeutig was die Kosten- und Qualitätseffekte des Wettbewerbs im Gesundheitswesen betrifft. Die einfache Forderung nach „mehr Wettbewerb“ wird somit der Komplexität des Gesundheitswesens nicht gerecht.

Schlüsselwörter: Gesundheitswesen, Wettbewerb, Krankenkassen, freie Arztwahl

JEL-Codes: I11, I18, D61

* Für Kommentare zur ersten Version dieses Textes danke ich Niels Krap und PD Dr. Joachim Wilde. 
IWH 


\section{Grenzen des Wettbewerbs im Gesundheitswesen}

\section{$1 \quad$ Einleitung}

Bei der Diskussion über Reformen in der deutschen gesetzlichen Krankenversicherung dominieren unter Gesundheitsökonomen Vorschläge zur Verstärkung des Wettbewerbs. Mit der Einführung der freien Wahl der Krankenkasse wurde 1997 ein Wettbewerb der Kassen um Versicherte eingeführt. Die Kassen verfügen jedoch nur über wenige Wettbewerbsinstrumente, denn der Leistungskatalog ist weitgehend vorgegeben und die Verträge mit den Leistungserbringern werden fast immer zentral ausgehandelt.

Vorschläge, den Wettbewerb zwischen den Krankenkassen zu verstärken, knüpfen vielfach an Enthovens Konzept des „Managed Competition“ an, bei dem die Versicherten eine Auswahl an Versicherungen und die Krankenversicherungen für ihr Versorgungsangebot vielfältige Gestaltungsrechte haben und bei dem der Wettbewerb durch ein System von Regeln gegen Gefahren des Marktversagens zu schützen ist.1 Können die Krankenkassen mit Ärzten, Krankenhäusern und anderen Leistungserbringern selektiv und individuell Versorgungsverträge schließen, dann erhalten sie dadurch die Möglichkeit, sich durch ihr jeweiliges Angebot bei den Versicherten gegenüber anderen Kassen zu profilieren. Zugleich eröffnet dies den Wettbewerb der Leistungserbringer um Verträge mit Krankenkassen, sodass sich der zwischen den Kassen herrschende Wettbewerbsdruck auf die Ebene der Leistungserbringung überträgt. Der Wettbewerb der Leistungserbringer um Verträge mit Kassen motiviert zu niedrigeren Kosten und besserer medizinischer Qualität. Solche Schritte zur Stärkung des Wettbewerbs werden von vielen Gesundheitsökonomen befürwortet und spielen in der gesundheitsökonomischen Literatur eine wichtige Rolle. ${ }^{2}$ Im Mittelpunkt aktueller wissenschaftlicher Empfehlungen zur Weiterentwicklung der gesetzlichen Krankenversicherung steht damit die Intensivierung des Wettbewerbs auf zwei Feldern: des Wettbewerbs der Leistungserbringer um Verträge mit Krankenkassen und des Wettbewerbs der Kassen um Versicherte.

Daneben besteht jedoch ein drittes Wettbewerbsfeld: der Wettbewerb der Leistungserbringer um Patienten. Obwohl dieser gelegentlich auch Erwähnung findet ${ }^{3}$, wird seine

1 Vgl. Enthoven (1993).

2 Vgl. zum Beispiel Breyer, Franz, Homburg, Schnabel und Wille (2004), S. 109 f.; Cassel (2005), S. 254 ff.; Cassel, Ebsen, Greß, Jacobs, Schulze und Wasem (2006), S. 25; Jacobs und Schulze (2004), S. 92; Sachverständigenrat zur Begutachtung der Entwicklung im Gesundheitswesen (2005), Ziffern 59-61; Wille (1999), S. 125-132; Wissenschaftlicher Beirat beim Bundesministerium für Wirtschaft und Technologie (2006), Ziffer 16.

3 Zum Beispiel bei Cassel, Ebsen, Greß, Jacobs, Schulze und Wasem (2006), S. 23 f. 
Bedeutung für die Effizienz im Gesundheitswesen vernachlässigt. Die meisten Reformvorschläge zielen allein auf die Verstärkung des Wettbewerbs in den anderen Wettbewerbsfeldern.

Im Folgenden wird argumentiert, dass der Wettbewerb der Leistungserbringer um Patienten ein wichtiger Antrieb zur Steigerung der medizinischen Qualität ist. Die These des Textes ist, dass er aber mit den beiden anderen Wettbewerben im Konflikt steht. Ferner wird das Verhältnis der Wettbewerbsfelder zu der sozialpolitischen Zielsetzung einer vom Einkommen unabhängigen Gesundheitsversorgung geprüft. In diesem Text soll deutlich werden, dass angesichts des Konflikts zwischen verschiedenen Wettbewerbsfeldern im Gesundheitswesen die einfache Forderung nach mehr Wettbewerb uneindeutig ist und es nicht einfach um ein „Mehr oder Weniger“ an Wettbewerb gehen kann.

Im folgenden zweiten Kapitel werden die drei Wettbewerbsfelder in einem System der sozialen Krankenversicherung dargestellt und ihre Bedeutung für Kosten und Qualität der Gesundheitsversorgung theoretisch untersucht. Die Interaktion der drei Wettbewerbsfelder wird betrachtet und damit die These des Textes hergeleitet und diskutiert. Dabei wird von den existierenden Gesundheitssystemen abstrahiert und lediglich ein System der sozialen Krankenversicherung zu Grunde gelegt. Im dritten Kapitel wird geprüft, ob die theoretisch hergeleiteten Wirkungen des Wettbewerbs durch bisherige Erfahrungen und Studien belegt werden können. In den Schlussfolgerungen werden die wichtigsten Ergebnisse noch einmal zusammengefasst und wird ein kurzer Ausblick auf mögliche gesundheitspolitische Konsequenzen gegeben. 


\section{Funktionen und Wechselwirkungen von Wettbewerbs- feldern im Gesundheitswesen}

Das System der sozialen Krankenversicherung ist im Wesentlichen in dem Dreieck zwischen Versicherten/Patienten, Leistungserbringern (v. a. niedergelassene Ärzte und Krankenhäuser) und Krankenversicherungen (im Bereich der sozialen Krankenversicherung: Krankenkassen) organisiert. ${ }^{4}$ Entsprechend sind hier drei Felder des Anbieterwettbewerbs denkbar: Der Wettbewerb der Krankenkassen um die Versicherten, der Wettbewerb der Leistungserbringer um die Krankenkassen und der Wettbewerb der Leistungserbringer um die Patienten. Das System insgesamt steht zusätzlich unter dem politisch gesetzten Vorbehalt, allen Gesellschaftsmitgliedern unabhängig vom Einkommen Zugang zur medizinischen Versorgung zu garantieren.

\subsection{Wettbewerb zwischen Krankenkassen um Versicherte}

Ein Wettbewerb zwischen Krankenkassen um Versicherte findet statt, wenn die Versicherten sich ihre Kasse frei wählen können und zugleich die Kassen Entscheidungsfreiheiten hinsichtlich Leistungen und Beitragshöhe besitzen.

Neben der Beitragshöhe kommen dabei als Wettbewerbsinstrumente der Umfang und die Ausgestaltung des Leistungskatalogs, die Auswahl der unter Vertrag genommenen Leistungserbringer, spezielle Versorgungsformen sowie der Service der Krankenkasse selbst in Frage. Der Wettbewerb um Versicherte motiviert die Krankenkassen dazu, sich für ein präferenzkonformes Angebot, gute Qualität und niedrige Kosten in der Gesundheitsversorgung einzusetzen.

Haben die Kassen die Möglichkeit, ihren Leistungskatalog selbst zu bestimmen, dann können sie den Patienten entweder für höhere Prämien eine umfassendere Absicherung oder für niedrigere Prämien ein geringeres Leistungsniveau bieten. Da in einem System der sozialen Krankenversicherung ein Mindest-Leistungskatalog gesetzlich vorgegeben sein muss, können hier Variationen des Leistungsumfangs nur begrenzt Teil des Wettbewerbsverhaltens der Kassen sein.

Eine andere Variation des Leistungsangebots könnte darin bestehen, nur ausgewählte Leistungserbringer unter Vertrag zu nehmen. Indem die Kassen mit Leistungserbringern selektiv Verträge schließen, könnten sie sich durch die bei ihnen jeweils unter Vertrag stehenden Leistungserbringer profilieren, beispielsweise durch Exklusiv-Verträge mit den besten Ärzten.

4 Vgl. Cassel (2005), S. 254, Abb. 2; Cassel, Ebsen, Greß, Jacobs, Schulze und Wasem (2006), S. 23 ff. 
Besonders weit geht in dieser Richtung das Modell des Managed Care. Dabei verbinden die Krankenkassen die Finanzierungs- mit der Leistungserbringerfunktion. Sie bauen feste Vertragsbindungen mit ausgewählten Ärzten und Krankenhäusern auf und bieten ihren Mitgliedern nicht nur die reine Kostenübernahme, sondern ein in sich stimmiges Gesamtkonzept von Leistungen und Versorgungsformen, das sich vom Angebot anderer Kassen unterscheidet.

Managed Care-Modellen wird neben ihrer Rolle im Wettbewerb auch der Vorteil zugeschrieben, eine bessere Koordination zwischen Leistungserbringern zu ermöglichen. Die Arbeit von Hausärzten, Fachärzten, Krankenhäusern und ggf. weiteren Gesundheitsdienstleistern kann unter dem Dach der Versicherung besser aufeinander abgestimmt werden als dies bei unkoordinierter freier Arztwahl des Patienten der Fall ist. Die Informationsflüsse zwischen den Leistungserbringern können optimiert werden, teure Doppeluntersuchungen werden vermieden.

\subsection{Wettbewerb zwischen Leistungserbringern um Krankenkassen}

Soll der Kassenwettbewerb zu Kostensenkung und Qualitätssteigerung beitragen, bedarf es des Wettbewerbs der Leistungserbringer um Krankenkassen. Dieser kann zu Stande kommen, wenn die Kassen die Möglichkeit besitzen, zwischen Leistungserbringern auszuwählen und ggf. einzelne Leistungserbringer auszuschließen, und wenn die Leistungserbringer mit den Kassen individuell über die Preise verhandeln können. Ein Wettbewerb der Leistungserbringer um Verträge mit Krankenkassen ist nicht nur ein Preis-, sondern auch ein Qualitätswettbewerb, da die Kassen auch ein Interesse daran haben, ihren Versicherten gute medizinische Qualität anzubieten.

\subsection{Wettbewerb zwischen Leistungserbringern um Patienten}

Die Freiheit der Arzt- und Krankenhauswahl bewirkt einen Wettbewerb zwischen den Leistungserbringern um Patienten. Ein Aspekt der freien Arztwahl ist der direkte Zugang der Patienten zu Fachärzten, durch den der Wettbewerb zusätzlich erhöht wird. Voraussetzung des Wettbewerbs ist ein Interesse der Leistungserbringer an vielen Patienten, welches u. a. dann begünstigt wird, wenn die Vergütung von der Zahl der behandelten Patienten abhängt. Da die Kosten meistens von Versicherungen oder dem Staat getragen werden, ist für Ärzte und andere Leistungserbringer im Wettbewerb um Patienten die Qualität der Versorgung das Wettbewerbsinstrument.5 Der Wettbewerb zwischen den Ärzten setzt somit einen Anreiz zur Steigerung der Qualität der Gesundheitsversorgung. Zugleich eröffnet die Abwanderungsmöglichkeit den Patienten einen

Der Preis könnte ein Wettbewerbsinstrument sein, wenn die Patienten ihn direkt zahlen müssten und er von den Ärzten festgelegt würde. Beides ist in der sozialen Krankenversicherung nicht der Fall, deshalb wird ein solcher Preiswettbewerb hier nicht genauer untersucht. 
Weg zur relativ schnellen Korrektur von therapeutisch nicht bewährten Ansätzen und Fehldiagnosen. Auch im Gesundheitswesen ist der Wettbewerb nicht nur Leistungsanreiz, sondern als Prozess von Versuch und Irrtum auch ein Entdeckungsverfahren. 6 Dabei muss der durch freie Arztwahl gewährleistete Ärztewettbewerb nicht zu einheitlichen Ergebnissen führen, sondern eröffnet die Möglichkeit zur individuell zugeschnittenen Versorgung. Angesichts der hohen Bedeutung, die das persönliche Verhältnis von Arzt und Patient für die Qualität der Behandlung hat, ist dies wichtig.

Wie sonst im Wettbewerb kann auch hier ein hohes Maß an Markttransparenz das Finden guter Lösungen erleichtern und den Leistungsanreiz erhöhen. Patientenorganisationen, Krankenkassen oder Ärztevereinigungen können Informationen über Leistungserbringer veröffentlichen, die den Patienten bei der Arztwahl helfen.

Der Wettbewerb zwischen den Leistungserbringern um Patienten ist zwar ein Anreiz zur Verbesserung medizinischer Qualität, er wirkt jedoch nicht als Anreiz zur Kosteneinsparung, wenn (wie in den sozialen Versicherungssystemen der Industrieländer) die Kosten von Krankenversicherungen oder vom Staat getragen werden. Auch die oft bestehenden begrenzten Zuzahlungen bremsen zwar die Inanspruchnahme von Gesundheitsleistungen durch die Versicherten, bewirken aber keinen Preiswettbewerb der Leistungserbringer, da ihre Höhe zentral vorgegeben und nicht vom Leistungserbringer selbst bestimmt wird.

Gelegentlich wird kritisiert, dass die freie Wahl der Leistungserbringer dazu beiträgt, dass in Fällen, in denen an Diagnose und Therapie mehrere Dienstleister (z. B. Hausarzt, Facharzt und Krankenhaus) beteiligt sind, diese zu schlecht auf einander abgestimmt handeln, wenn die Auswahl vom Patienten freihändig erfolgt. Hausarztmodelle, bei denen der Hausarzt alle notwendigen Überweisungen vornimmt und die Gesundheitsinformationen eines Patienten zentral verwaltet, oder erweiterte Koordinationsaufgaben der Versicherung werden als Abhilfe vorgeschlagen.

\subsection{Interaktionen der drei Wettbewerbsfelder und der sozialpoliti- schen Zielsetzung}

Die drei genannten Wettbewerbsfelder stehen in enger Interaktion. Der Wettbewerb zwischen Krankenkassen erfordert es, dass die Kassen Spielräume haben, in denen sie ihr Leistungsangebot preislich und qualitativ variieren können. Diesen Spielraum haben sie insbesondere dann, wenn sie mit Leistungserbringern selektiv und unterschiedlich ausgestaltete Verträge abschließen können, wodurch der Wettbewerb der Leistungserbringer um die Kassen entsteht. Umgekehrt ist der Wettbewerb der Leistungserbringer um die Krankenkassen nur dann ein wirksames Mittel zur Steigerung der Effizienz, 
wenn die Kassen ein Interesse an niedrigen Kosten und guter Qualität haben, ein Interesse, das vor allem dann vorliegt, wenn die Kassen ihrerseits im Wettbewerb um die Versicherten stehen, die sie durch gute Qualität zu niedrigen Preisen für sich gewinnen wollen. Der Wettbewerb der Leistungserbringer um Kassen und der Kassen um Versicherte stehen in einem engen komplementären Verhältnis. Viele Gesundheitsökonomen empfehlen, den Wettbewerb in diesen beiden Feldern zu verstärken. ${ }^{7}$

Die Stärkung des Wettbewerbs zwischen Leistungserbringern durch selektive Verträge der Krankenkassen mit ihnen impliziert, dass die Krankenkassen die Möglichkeit haben, mit einzelnen Leistungserbringern auch keinen Vertrag abzuschließen. Für die Versicherten bedeutet dies eine Begrenzung der Arzt- und Krankenhauswahl auf die bei der eigenen Kasse unter Vertrag stehenden Leistungserbringer.8 Ein Arztwechsel kann so u. U. nur bei vorangegangenem Wechsel der Kasse erfolgen. Da aber der Wechsel der Krankenkasse mit höheren Transaktionskosten verbunden ist als der Wechsel des Arztes, ist im Krankheitsfall die freie Arztwahl eingeschränkt. ${ }^{9}$ Insbesondere in ländlichen Regionen mit geringer räumlicher Ärztedichte oder im Bereich spezialisierter Fachärzte kann die Einschränkung der freien Arztwahl dazu führen, dass einzelne Ärzte gegenüber den Patienten faktisch als Monopolisten auftreten. Dies gilt vor allem bei Managed Care-Modellen, in denen sogar bewusst Ärzte bzw. Ärzteverbünde als Monopolisten eingesetzt werden. Der Wettbewerb der Leistungserbringer um Patienten und damit beispielsweise die Möglichkeit, Fehldiagnosen durch Einholung einer zweiten Meinung schnell zu korrigieren oder Ärzte durch die Patienten selbst auf ihre Qualität hin zu vergleichen, wäre eingeschränkt.

Die Auswahl eines den individuellen Bedürfnissen entsprechenden Arztes wird in einem solchen System behindert. Denn die Suche und das Finden des passenden Arztes erfolgt nicht bei Eintritt in die Krankenkasse, sondern erst im Krankheitsfall - und dann notgedrungen nach dem Verfahren von Versuch und Irrtum. Andererseits kann ein Wechsel der Krankenkasse dazu führen, dass zugleich der Arzt gewechselt werden muss, wodurch z. B. ein gewachsenes Vertrauensverhältnis zwischen Arzt und Patient zerschnitten wird. Alternativ kann dies bedeuten, dass gerade deshalb der Wechsel der Krankenkasse unterbleibt, auch wenn der Versicherte mit deren Service und Beitragssatz unzufrieden ist.

Das Prinzip selektiver Verträge der Kassen mit den Ärzten und damit der verstärkte Wettbewerb der Ärzte um Verträge mit Krankenkassen korrespondiert also mit einer

7 Vgl. die Literaturangaben in Anm. 2.

8 Vgl. Wille (1999), S. 130; Wissenschaftlicher Beirat beim Bundesministerium für Wirtschaft und Technologie (2006), Ziffer 36.

9 Im Falle multimorbider Patienten, die bei mehreren Ärzten verschiedener Fachrichtungen in Behandlung sind, kann sich zusätzlich das Problem ergeben, dass die Fachärzte des Vertrauens nicht alle bei derselben Krankenkasse unter Vertrag sind. 
Einschränkung des Wettbewerbs der Ärzte um die Gunst der Patienten, also einer Einschränkung des hier stattfindenden Qualitätswettbewerbs.

Der Wettbewerb der Leistungserbringer um die Kassen ist aber auch ein Qualitätswettbewerb, sodass mit Einschränkung der freien Arztwahl durch Einführung eines Systems selektiver Verträge lediglich eine Form des Qualitätswettbewerbs durch eine andere ersetzt wird, aber zusätzlich der Wettbewerb um niedrige Kosten gestärkt wird. Somit stellt sich die Frage, ob der Wettbewerb zwischen Ärzten um Krankenkassen oder der Wettbewerb zwischen Ärzten um Patienten wirksamer zur Steigerung medizinischer Qualität beiträgt.

Die Wahl der Krankenkasse erfolgt typischerweise zu einer Zeit, in der man gesund ist und Beiträge zu zahlen hat, und in der folglich der Beitragssatz für die Wahl der Kasse stärker ins Gewicht fällt als die - ohnehin im Voraus und in Unkenntnis künftiger Krankheiten schwer messbare - angebotene medizinische Qualität. Umgekehrt erfolgt die Wahl des Arztes im Krankheitsfall und dann unter qualitativen Aspekten. Deshalb wird im Wettbewerb der Kassen der preisliche Aspekt verglichen mit der medizinischen Qualität tendenziell die größere Rolle spielen. So wie bei freier Arztwahl einem intensiven Qualitätswettbewerb ein fehlender Preiswettbewerb gegenübersteht, zeichnet sich der Wettbewerb der Krankenkassen durch einen verstärkten Preiswettbewerb bei einem schwächeren Qualitätswettbewerb aus.

Gelegentlich wird argumentiert, die Krankenkassen seien besser als die Patienten über die Qualität der Leistungserbringer informiert.10 So können sie über umfangreiche Daten verfügen, welche Schlussfolgerungen über die Qualität, Spezialgebiete und Erfahrungen von Leistungserbringern erlauben. Andererseits ist gerade bei Gesundheitsleistungen das persönliche Vertrauensverhältnis zwischen Patient und Arzt wesentlich, sodass in die Qualität des Produkts auch subjektive Aspekte eingehen, die sich der Messung durch eine Institution grundsätzlich entziehen. Der Wettbewerb zwischen Leistungserbringern um Patienten dient nicht allein als Leistungsanreiz, sondern ebenso als Mittel, um die Auswahl zwischen verschiedenen Angeboten zu ermöglichen und so individuell passende und gerade nicht für alle einheitliche Lösungen zu finden. Außerdem wirkt er qualitätssteigernd indem er die schnelle Korrektur von Fehldiagnosen durch Abwanderung zu einem anderen Arzt erleichtert. Hinzu kommt, dass die Patienten die Qualität einer medizinischen Versorgung ganz direkt erleben, während die Informationen der Krankenkassen über die Qualität von Ärzten zwangsläufig indirekte Erhebungen darstellen. Allerdings haben solche Erhebungen den Vorteil, dass sie sich auf große Zahlen stützen.

Die Aussage, die Krankenkassen seien über die Qualität von Ärzten besser informiert als die Versicherten, ist folglich zu relativieren. Während die Kassen Zugang zu größe- 
ren Erhebungen haben können, kennen die Patienten das persönliche Vertrauensverhältnis und die Diagnose im Einzelfall. Dadurch besteht eine für die Frage der freien Arztwahl wesentliche Asymmetrie: Während die Krankenkassen den Patienten grundsätzlich Zugang zu ihren Erkenntnissen geben und so deren Arztwahl unterstützen können, ist umgekehrt für die Kassen eine Information über das persönliche Vertrauensverhältnis im individuellen Fall nicht verallgemeinerbar und höchstens von marginaler Bedeutung.

Man könnte argumentieren, dass der Wettbewerb der Ärzte um Krankenkassen stärker zur medizinischen Qualität beiträgt als der Wettbewerb der Ärzte um Patienten, weil eine Krankenkasse über eine größere Verhandlungsmacht verfügt als ein einzelner Patient. ${ }^{11}$ Die Drohung, eine Kasse mit vielen Versicherten als Vertragspartner zu verlieren, sei für einen Arzt wirksamer als die Drohung, einen einzelnen Patienten zu verlieren. Dem gegenüber könnte es jedoch sein, dass die Versicherten ihren Ärzten treuer sind als ihrer Krankenkasse. Schließlich besteht zum Arzt oft ein persönliches Vertrauensverhältnis, während die Krankenkasse im Kern meistens eine anonyme Abrechnungsstelle bleibt. In diesem Fall würde die Krankenkasse, die einen Arzt als Vertragspartner ausschließt, damit zugleich dessen Patienten als Versicherte verlieren.12

Insgesamt ist der Wettbewerb der Ärzte um Krankenkassen dem Wettbewerb der Ärzte um Patienten in Bezug auf den Anreiz zur Steigerung medizinischer Qualität nicht überlegen. Sind die Kassen (und auch andere Institutionen) bereit, ihre Informationen den Patienten zur Verfügung zu stellen, dann kann die freie Arztwahl der Patienten zum Qualitätswettbewerb deutlich mehr beitragen als ein System, in dem die Krankenkassen mit Ärzten selektiv Verträge abschließen.

Der Wettbewerb der Kassen um Versicherte und der Leistungserbringer um Kassen einerseits und der Wettbewerb der Leistungserbringer um Patienten andererseits stehen miteinander im Konflikt. Dabei sind die beiden ersten Wettbewerbe geeignet zur Kostendämpfung, da bei ihnen der Preiswettbewerb dominiert, ferner beinhalten sie auch einen Qualitätswettbewerb. Letzterer kann jedoch zu einem wirkungsvolleren Wettbewerb um gute Qualität führen. Es besteht hier somit ein Zielkonflikt zwischen Kostendämpfung und Qualitätssteigerung, dessen Entscheidung durch die Zulassung von Wettbewerb in den verschiedenen Feldern gesundheitspolitisch erfolgt.

In welchem Verhältnis stehen die Wettbewerbsfelder zu dem sozialpolitisch gesetzten Ziel einer vom Einkommen unabhängigen Gesundheitsversorgung für alle?

Der Kassenwettbewerb erfordert die Möglichkeit selektiver Verträge mit Ärzten. Als gut geltende Ärzte könnten dabei gegenüber den Krankenkassen höhere Vergütungen durch-

11 Für dieses Argument danke ich Alexander Kubis.

12 Bestätigt wird dies durch Erfahrungen in den Niederlanden, wo die Kassen von der Möglichkeit zu selektiven Verträgen kaum Gebrauch machen, vgl. Kapitel 3.3. 
setzen. Krankenkassen, die begehrte Ärzte unter Vertrag haben, müssten die höheren Kosten an die Versicherten weitergeben, sodass nur Versicherte, die zur Zahlung höherer Beiträge bereit und in der Lage sind, Zugang zu den Ärzten mit ,gutem Ruf“ erhalten. Umgekehrt werden Krankenkassen, die durch niedrige Beiträge für Geringverdiener interessant sind, angesichts des knapper bemessenen Budgets nur weniger begehrte und deshalb billigere Ärzte unter Vertrag nehmen können. Im Ergebnis würden teure Kassen auch die bessere Versorgung bieten, während billige Kassen auch nur Billig-Medizin im Angebot hätten. Das sozialpolitisch vorgegebene Ziel einer vom Einkommen unabhängigen guten medizinischen Versorgung wird durch den Kassenwettbewerb verletzt.

Doch auch wenn alle Krankenkassen mit allen Ärzten Verträge schließen, entsteht das Problem der Medizin nach Geldbeutel. Krankenkassen mit niedrigen Beiträgen (und deshalb ärmeren Mitgliedern) könnten den Ärzten nur niedrige Honorare bieten. Folglich wären ihre Mitglieder als Patienten eher unwillkommen. Auch in diesem Fall werden Geringverdiener benachteiligt. Der Wettbewerb von Leistungserbringern um Krankenkassen und der Krankenkassen um Versicherte steht im Konflikt mit der sozialpolitischen Zielsetzung.

Der Wettbewerb der Leistungserbringer um Patienten steht dagegen in keinem systematischen Konflikt mit dem sozialpolitisch gesetzten Ziel. Er vernachlässigt aber wie gesagt das Ziel der Kostenbegrenzung. 


\section{Empirische Befunde zur Wirkung von Kassen- und Ärztewettbewerb}

In diesem Kapitel wird überblicksartig geprüft, ob die theoretische These dieses Textes in vorhandenen empirischen Befunden bestätigt wird. Zuerst wird ein kurzer Überblick gegeben, inwieweit in bestehenden Gesundheitssystemen Wettbewerb zugelassen wird (3.1). Dann wird auf die Evidenz zu den Folgen des Wettbewerbs zwischen Krankenkassen für die Kosten des Gesundheitswesens eingegangen (3.2). Vorhandene Erfahrungen mit möglichem Wettbewerb zwischen Leistungserbringern um Versicherungen werden behandelt (3.3). Im letzten Teil (3.4) geht es um die Folgen des Wettbewerbs zwischen Leistungserbringern um Patienten für die Qualität.

\subsection{Wettbewerb in den bestehenden Gesundheitssystemen ${ }^{13}$}

Wettbewerb zwischen einzelnen Versicherungen ist im Bereich der sozialen Krankenversicherung international eher unüblich. So ist in vielen Ländern die Krankenversicherung Teil der allgemeinen Staatsverwaltung (so in Großbritannien) oder es besteht keine Wahl der Krankenkasse. Die Freiheit der Wahl zwischen Krankenkassen gibt es in Belgien, den Niederlanden, Deutschland, der Schweiz, Tschechien und Israel. In diesen Ländern konkurrieren die Kassen um Versicherte. Dabei verfügen sie jedoch nur in eingeschränktem Umfang über Wettbewerbsinstrumente. Der Beitragssatz kann nur in der Schweiz, den Niederlanden und Deutschland von den Kassen eigenständig festgelegt und damit als Wettbewerbsinstrument genutzt werden. Der Leistungskatalog ist weitgehend zentral festgelegt. Meistens haben die Kassen alle Ärzte unter Vertrag und wird die Ärztevergütung zentral festgelegt. Selektive Verträge mit Leistungserbringern können die Kassen nur in den Niederlanden und Israel schließen. ${ }^{14}$ Folglich ist der Wettbewerb sehr begrenzt. Allein im Bereich von Service und Verwaltung können die Krankenkassen selbst entscheiden und versuchen, Kosteneinsparungen und Qualitätsverbesserungen zu erzielen.

Der Wettbewerb zwischen Leistungserbringern um Patienten erfordert das Recht der Patienten auf freie Wahl des Leistungserbringers. In einigen Ländern sind die freie Arztund Krankenhauswahl und der direkte Zugang auch zu Fachärzten garantiert.15 Allerdings setzt die Gesundheitspolitik in einigen Ländern finanzielle Anreize zum Verzicht

13 Die folgenden Angaben beruhen auf Angaben in: European Observatory (verschiedene Jahre) und Europäische Kommission (2007).

14 Ausnahmen gibt es in Deutschland und der Schweiz bei Versicherten, die sich bei Beitragsermäßigung für besondere Versorgungsformen ohne freie Arztwahl entscheiden.

15 So in Deutschland, Belgien, Luxemburg, der Schweiz und Schweden. 
auf häufige Wechsel des Arztes. 16 In vielen Ländern wird der Arztwechsel erschwert, indem die Versicherten sich bei einem Hausarzt fest registrieren lassen müssen 17 oder der Zugang zum Facharzt nur nach einer Überweisung des Allgemeinmediziners gewährt wird (Primärarztmodell)18. Am weitesten geht die Einschränkung der freien Arztwahl bei Hausarztmodellen und Managed Care-Modellen (insbesondere den Health Maintenance Organizations, HMO), bei denen bewusst die freie Arztwahl aufgehoben wird. Letztere sind insbesondere in privaten Krankenversicherungen der USA und als begünstigte Wahltarife in der Schweiz verbreitet.

\subsection{Kassenwettbewerb zur Kostendämpfung}

Zwar haben die beiden Länder, die auf ihren Krankenversicherungsmärkten am stärksten auf Wettbewerb zwischen Versicherungen setzen - die USA und die Schweiz - die weltweit höchsten Gesundheitsausgaben. Allerdings würde eine verlässliche Aussage über die Kostenwirkungen des Wettbewerbs zwischen Krankenversicherungen die Einbeziehung wichtiger Kontrollvariablen erfordern, z. B. den Leistungsumfang, die Morbidität der Bevölkerung usw.

Im bisherigen Wettbewerb zwischen Krankenkassen in Ländern wie Deutschland oder den Niederlanden mangelt es den Krankenkassen an Möglichkeiten, sich durch kostensenkende Maßnahmen am Markt zu profilieren. Deshalb stellt sich die Frage, ob der Wettbewerb dort kostendämpfend wirkte, wo dem keine gesetzlichen Vorgaben entgegenstehen: bei den Verwaltungsausgaben der Krankenversicherung.

Ein internationaler Vergleich der Verwaltungskosten leidet unter einem Mangel an vergleichbaren Daten. So ist in internationalen Statistiken nicht sichergestellt, dass für alle Länder dieselbe Definition von Verwaltungsausgaben zu Grunde gelegt wird. Außerdem hängen die Verwaltungsausgaben auch von der Komplexität des Gesundheitswesens allgemein ab, sodass Krankenversicherungen z. B. in Ländern mit komplizierten Abrechnungsverfahren unabhängig vom Grad des Wettbewerbs vermutlich auch höhere Verwaltungskosten aufweisen. Aussagekräftiger sind deshalb hier Vergleiche für die selben Länder im Zeitverlauf, insbesondere für Länder, in denen der Wettbewerb zwischen Krankenkassen erst vor wenigen Jahren eingeführt wurde.

Betrachtet man die Entwicklung des Anteils der Verwaltungskosten im Zeitverlauf, dann ergibt sich ein gemischtes Bild (vgl. Abbildung). In der Schweiz ging der Anteil

16 In Norwegen und Frankreich ist der direkte Zugang zum Facharzt nur bei erhöhten Zuzahlungen möglich. In Deutschland und der Schweiz werden besondere Versorgungsformen mit beschränkter Arztwahl gefördert.

17 Zum Beispiel in Großbritannien, Norwegen, Portugal, Polen, Tschechien, der Slowakei, Ungarn.

18 Zum Beispiel in Großbritannien, Irland, den Niederlanden, Spanien, Italien, Polen oder Ungarn. 
der Verwaltungsausgaben an den Gesamtausgaben der sozialen Krankenversicherung nach Inkrafttreten des Krankenversicherungsgesetzes 1996 zurück. Hier gab es jedoch bereits zuvor Wettbewerb zwischen den Versicherern. Möglicherweise kann der Rückgang der Verwaltungsausgaben damit erklärt werden, dass die Versicherer seit 1996 bei Aufnahme von neuen Versicherten keine Gesundheitsprüfungen mehr durchführen, da ihnen (im Gegensatz zu früher) die Erhebung risikoabhängiger Prämien verboten ist. In den Niederlanden sank der Anteil der Verwaltungsausgaben nach Einführung des Kassenwettbewerbs. In Deutschland dagegen kam es nach der Einführung der Wahlfreiheit 1997 sogar zu steigenden Verwaltungsausgaben.

Abbildung:

Anteil der Verwaltungsausgaben in Ländern mit freier Wahl der Krankenkasse

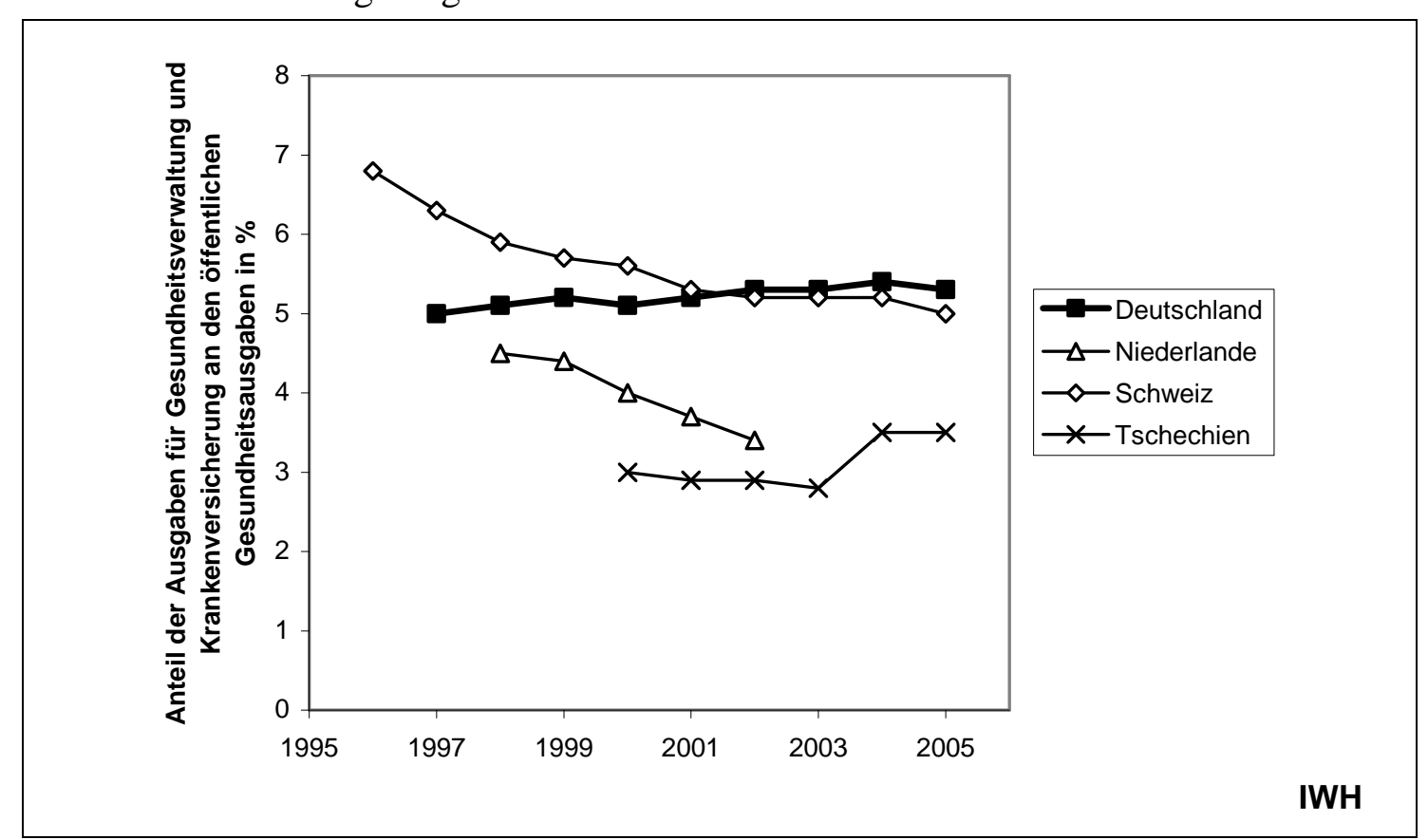

Quelle: OECD Gesundheitsdaten 2007.

Der Anstieg der Verwaltungskosten in Deutschland wird damit begründet, dass die Strukturen des Gesundheitswesens immer komplizierter und damit die Verwaltungsaufgaben umfangreicher werden, z. B. durch die Einführung der DRG-Vergütung der Krankenhäuser oder der neuen Versorgungsformen (integrierte Versorgung, Disease Management-Programme usw.). ${ }^{19}$ Auch könnte es sein, dass Kassen, die im Zuge des Wettbewerbs Mitglieder verlieren, ihre Kapazitäten langsamer abbauen können als die von der Zuwanderung profitierenden Kassen neue Kapazitäten aufbauen.20 Ferner

19 Vgl. Ulrich (2004), S. 119, 121.

20 Vgl. Deutsche Bundesbank (2004), S. 22 f. 
könnte der Wettbewerb neue Kosten für Marketingmaßnahmen zur Abwerbung von Versicherten anderer Kassen verursacht haben. Die erhofften kostendämpfenden Effekte des Wettbewerbs (etwa Einsparungen infolge effizienter organisierter Verwaltungsabläufe) waren offenkundig zu schwach, um die kostensteigernden Impulse auszugleichen.

Insgesamt ist die Evidenz für einen kostensenkenden Druck des Wettbewerbs im internationalen Vergleich schwach, leidet aber daran, dass aussagefähige Tests nur schwer durchführbar sind. Das Beispiel der Entwicklung der Verwaltungskosten in den Niederlanden stützt die Hypothese, dass vermehrter Kassenwettbewerb kostensenkend wirkt. Andere Beispiele werden durch länderspezifische Faktoren und Ereignisse so stark geprägt, dass sich der Effekt des Wettbewerbs nicht ohne weiteres herausfiltern lässt.

\subsection{Kostenwettbewerb der Leistungserbringer um Krankenkassen}

Es kann sein, dass die Ärzte auch bei der Möglichkeit freier Verträge mit Krankenkassen kaum in einen intensiven Preiswettbewerb eintreten würden. So wären die Transaktionskosten von einzeln durchzuführenden Verhandlungen zwischen Krankenkassen und Ärzten sehr hoch. Wahrscheinlich schließen sich auch unter diesen Bedingungen Ärzte zu Verbänden zusammen, die zentrale Vergütungsverhandlungen durchführen.21

In Ländern, die selektive Verträge der Krankenversicherungen mit Leistungserbringern zulassen, hat sich gezeigt, dass ein Wettbewerb zwischen Ärzten um die Gunst von Krankenkassen oft auch dann unterbleibt, wenn er gesetzlich zugelassen ist. In den Niederlanden blieben die Möglichkeiten der Kassen, einzelne Hausärzte auszuschließen oder unterschiedlich hohe Honorare zu vereinbaren, weitgehend ungenutzt. 22 Die Ursache dürfte darin liegen, dass den meisten Versicherten die Bindung zu ihrem Hausarzt wichtiger ist als zu ihrer Krankenkasse, sodass die Krankenkasse mit dem Ausschluss eines Arztes zugleich dessen Patienten als Versicherte verlieren würde. In Israel, wo vielfach Ärzte bei den Polikliniken der Krankenkassen angestellt sind, werden die Ärzte von ihrer Gewerkschaft zentral vertreten, sodass ebenfalls der Wettbewerb der einzelnen Ärzte um die Krankenkassen schwach bleibt. Die Beispiele belegen, dass sich ein Wettbewerb in diesem Gebiet nur schwer gegen den Willen und die Organisationsfähigkeit der Beteiligten durchsetzen lässt.

21 Vgl. Sachverständigenrat (2005), Ziffer 83. Bestätigt wird dies in neuester Zeit in der Region Kassel. Hier haben im Oktober 2007 Ersatzkassen erstmals eine durch die Gesundheitsreform neu geschaffene Möglichkeit genutzt und die ambulante Versorgung von Versicherten (die freiwillig teilnehmen) unter Ärzten und Gesundheitszentren ausgeschrieben. Darauf kam es am 10. November 2007 zur Gründung einer Ärztegenossenschaft, an der sich sofort über 500 Mediziner der Region beteiligten, in der sich die Mitglieder dazu verpflichten, Einzelverträge mit Kostenträgern nur nach Genehmigung der Genossenschaft abzuschließen.

22 Vgl. Greß, Okma und Hessel (2001), S. 16, 20. 
Stärkerer Wettbewerb von Leistungserbringern herrscht insbesondere bei Managed Care-Organisationen, wie den HMO, die in den USA und der Schweiz verbreitet sind und in denen die Versicherung Vertragsbeziehungen nur zu ausgewählten Leistungserbringern hat. Die kostendämpfende Wirkung der HMO verglichen mit herkömmlichen Versicherungen wird kaum in Frage gestellt und in verschiedenen Studien für die USA überwiegend bestätigt. 23 Allerdings ist $\mathrm{zu}$ bedenken, dass tendenziell gesündere und preisbewusste Versicherte sich in HMO versichern, während Kranke diese eher verlassen, sodass niedrigere Ausgaben bereits allein aus der Versichertenstruktur der HMO resultieren.24 In ihrer Studie über die schweizerischen HMO versucht die Prognos AG, solche Effekte herauszurechnen und kommt dennoch zu dem Ergebnis, dass HMO gegenüber herkömmlichen Versicherungsformen einen Kostenvorteil von etwa 30 bis 35 Prozent aufweisen.25 Die engere Integration von Leistungserbringer und Kostenträger in der HMO ändert die Anreizstruktur in Richtung Kostendämpfung.

Ursache niedriger Kosten ist wenigstens teilweise eine geringere Nutzung teurer medizinischer Verfahren, wie mehrere Studien für die USA und die Schweiz bestätigen.26 Dies zeigt, dass die HMO ihren Kostenvorteil nicht oder nicht nur durch niedrigere Preise, sondern mindestens teilweise durch Einsparung von eingesetzten Ressourcen erzielen. Möglicherweise ist dies auch auf den Vorteil einer besseren Koordination zwischen Leistungserbringern innerhalb der HMO zurückzuführen, wodurch bei Beteiligung mehrerer Leistungserbringer (Hausarzt, Facharzt, Krankenhaus) teure Doppeluntersuchungen vermieden werden.

\subsection{Wettbewerb der Leistungserbringer um Patienten und medizini- sche Qualität}

Verschiedene internationale Patientenbefragungen geben Hinweise auf die Wirkung des Wettbewerbs zwischen Leistungserbringern um Patienten auf die Qualität der Versorgung. Die EUROCOM-Studie zum Verhältnis von Hausarzt und Patient von 1996/99 bestätigt die große Bedeutung des Wettbewerbs um Patienten als Leistungsanreiz für Ärzte. In Ländern mit ungehindertem Zugang der Patienten zu allen Ärzten (Deutschland, Belgien, Schweiz) nahmen sich demnach Hausärzte für ihre Patienten mehr Zeit als in Ländern mit Primärarztsystemen (Großbritannien, Spanien, Niederlande). Außerdem erwies sich in Regionen mit höherer Ärztedichte (und damit stärkerem Wettbewerb) die Gesprächsdauer als signifikant länger.27 Auch eine andere Befragung (die

23 Vgl. Miller und Luft (1997), S. 11; Miller und Luft (2002), S. 75.

24 Vgl. Klingenberger (2002), S. $10 \mathrm{f}$.

25 Vgl. Baur und Stock (2002), S. 142; ähnlich Perneger, Etter und Schaad (1995).

26 Vgl. Miller und Luft (1997), S. 10; Miller und Luft (2002), S. 75; Baur und Stock (2002), S. 143.

27 Vgl. Bahrs (2003). 
EUROPEP-Studie) bestätigt, dass Patienten in Ländern ohne Primärarzt-System (Deutschland, Belgien, Schweiz) mit ihren Hausärzten zufriedener sind als Patienten in Primärarzt-Ländern wie Großbritannien, Norwegen, den Niederlanden oder Dänemark. 28

Auch für Krankenhäuser ermitteln Coulter/Cleary in einer Studie für fünf Länder, dass die Patientenzufriedenheit in Großbritannien am geringsten ist, wo die Patienten bei der Krankenhauswahl am wenigsten frei sind. Dagegen schneiden Deutschland, Schweden, die Schweiz und die USA besser ab.29 Der Wettbewerb der Leistungserbringer um Patienten steigert die Patientenzufriedenheit.

Besonderes Interesse haben die Folgen der Managed Care-Organisationen für die medizinische Qualität gefunden. In diesen Organisationen, insbesondere den HMO, ist die freie Arztwahl der Patienten und damit der Wettbewerb der Leistungserbringer um Patienten eingeschränkt. Ist die Argumentation aus Kapitel 2.4 korrekt, dann müssten die HMO (bei niedrigeren Kosten) eine schlechtere medizinische Qualität aufweisen.

Für die Schweiz zeigt eine Studie, dass sich nach Einführung der HMO zwar keine messbare Verschlechterung des Gesundheitszustandes einstellte, aber die Patienten deutlich unzufriedener wurden. 30 Eine andere Schweizer Patientenbefragung ergab dagegen keinen Unterschied in der allgemeinen Zufriedenheit, allerdings einen schlechteren Zugang der Patienten zu Gesundheitsleistungen. 31

Hellinger liefert einen Überblick über fünf Studien zu HMO in den USA. Von diesen kommen drei zu dem Ergebnis, dass HMO insgesamt hinsichtlich der Ergebnisse bei der Behandlung von Krankheiten ähnlich abschneiden wie traditionelle Versicherungen. Allerdings sind die Ergebnisse bei Versicherten mit niedrigem Einkommen deutlich schlechter als bei traditionellen Versicherungen. Die zwei anderen Studien belegen, dass bei bestimmten Krankheiten die HMO gleich gute, bei anderen Krankheiten aber schlechtere Ergebnisse als andere Versicherungsformen erzielen. Ein Überblick über acht Studien, die auf der Grundlage von Befragungen Zufriedenheit und Zugang der Patienten zu ärztlicher Betreuung evaluieren, zeigt, dass Managed Care-Versicherte im Ganzen deutlich weniger zufrieden sind als herkömmlich Versicherte und größere Schwierigkeiten beim Zugang etwa zu fachärztlicher Versorgung haben.32

28 Vgl. Grol, Wensing u. a. (2000), S. 886. Davon weicht lediglich das Ergebnis für Schweden ab.

29 Vgl. Coulter und Cleary (2001).

30 Vgl. Perneger, Etter und Schaad (1995).

31 Vgl. Baur und Stock (2002), S. 147 f.

32 Vgl. Hellinger (1998). 
In zwei Überblicken über die Ergebnisse von 37 bzw. 79 Studien zu den Folgen von Managed Care, vor allem HMO, für die medizinische Versorgung zeigen Miller und Luft, dass die Ergebnisse hinsichtlich messbarer Qualität gemischt ausfallen:33 Eine jeweils ähnliche Zahl von Studien ermittelt für HMO bessere und schlechtere Ergebnisse im Vergleich zu herkömmlichen Versicherungsformen. Allerdings zeigen die hier einbezogenen Studien eine schlechtere Qualität der HMO bei der Versorgung chronisch kranker und alter Menschen. Auch bestätigen die hier einbezogenen Studien, dass der Zugang zur medizinischen Versorgung bei HMO schlechter und dass die Patientenzufriedenheit fast durchgängig geringer ist. Bessere Ergebnisse als andere Versicherungsformen erzielen HMO nur im Bereich der Vorsorge.

Insgesamt belegen die Ergebnisse, dass die mit Managed Care verbundenen Einschränkungen der freien Arztwahl bei den objektiv messbaren Gesundheitszuständen nicht generell zu schlechteren Ergebnissen führen. Schlechter sind jedoch die qualitativen Ergebnisse für besonders verletzliche Bevölkerungsgruppen wie Geringverdiener, Alte und chronisch Kranke. Außerdem zeigt sich, dass die Versorgungsstrukturen ohne freie Arztwahl zu einer geringeren Patientenzufriedenheit führen.

Das theoretisch hergeleitete Ergebnis, wonach die Einschränkung der freien Arztwahl zu schlechterer medizinischer Qualität führt, kann somit nur teilweise bestätigt werden. Eine Erklärung könnte darin bestehen, dass Managed Care-Organisationen zwar den Wettbewerb der Leistungserbringer um Patienten beschränken, aber eine bessere Koordination der Arbeiten verschiedener Leistungserbringer erlauben. 


\section{Schlussfolgerungen}

Das Ziel, im Gesundheitswesen sinkende Kosten und steigende Qualität generell durch Wettbewerb zu erreichen, stößt auf den grundsätzlichen Konflikt zwischen Kassenwettbewerb und Ärztewettbewerb um Kassen einerseits und Ärztewettbewerb um Patienten andererseits. Erstere tragen überwiegend zur Kostendämpfung, letzterer allein zur Qualitätsverbesserung bei. Dieser theoretische Befund wird empirisch teilweise bestätigt, allerdings sind die empirischen Ergebnisse weniger eindeutig.

So spricht für den kostensenkenden Effekt des Wettbewerbs zwischen Krankenkassen und zwischen Ärzten um Krankenkassen vor allem die belegte Kostensenkung in den Managed Care-Organisationen. Diese kann vermutlich auch mit der besseren Koordinierung der Gesundheitsleistungen in diesen Organisationen erklärt werden.

Gut belegt ist, dass Einschränkungen der freien Arztwahl zu geringerer Betreuungsintensität und bei den Patienten zu gesteigerter Unzufriedenheit führen. Außerdem gibt es Hinweise, dass bei eingeschränkter Arztwahl die medizinische Qualität bei Problemgruppen, wie Geringverdienern, alten und chronisch kranken Menschen, schlechter ausfällt. Darüber hinaus ließ sich bei Maßen für Gesundheitszustände bislang aber empirisch kein Qualitätsunterschied zwischen Managed Care-Modellen mit beschränkter Arztwahl und herkömmlichen Modellen mit freier Arztwahl robust belegen. Ob dies am Wettbewerb oder an der Überlegenheit der Managed Care-Modelle hinsichtlich der Koordination zwischen verschiedenen Leistungserbringern liegt, müsste zusätzlich untersucht werden.

Außerdem stehen die Modelle eines Wettbewerbs der Krankenkassen in der Tendenz im Konflikt mit dem sozialpolitisch gesetzten Ziel einer vom Einkommen unabhängigen Gesundheitsversorgung für alle.

Die einfache Forderung nach „mehr Wettbewerb im Gesundheitswesen“ durch mehr Wettbewerb zwischen Krankenkassen und zwischen Ärzten um Verträge mit Krankenkassen wird der Komplexität des Gesundheitswesens nicht gerecht. Wettbewerb ist für die Qualität im Gesundheitswesen wichtig, darf aber gerade nicht bedeuten, dass die Versicherten ihre Konsumentensouveränität an eine Versicherung als Sachwalter abtreten. Stattdessen sind vielmehr detailorientierte Lösungen zu suchen, die die Vorteile verschiedener Wettbewerbsmodelle kombinieren.

Modellen mit beschränkter Arztwahl (Managed Care, Hausarztmodelle) wird insbesondere der Vorteil zugeschrieben, die Koordination zwischen verschiedenen Leistungserbringern wie Hausarzt, Facharzt und Krankenhaus zu verbessern, dadurch teure Doppeluntersuchungen $\mathrm{zu}$ vermeiden und einen Beitrag zur medizinischen Qualität zu leisten. Es wäre anzustreben, diese Vorteile auch ohne Einschränkung der freien Arztwahl zu erreichen. Zu denken wäre an organisatorische Weiterentwicklungen, wie freiwillig wählbare medizinische Netzwerke oder die stärkere Einbeziehung von Krankenhäusern in 
die ambulante Versorgung. Sinnvoll wäre eine stärkere Einbindung des Patienten selbst in die Koordination seiner empfangenen Gesundheitsleistungen, z. B. durch umfassende Information, entsprechend dem Leitbild des mündigen Patienten.

Informationen zu Qualität und Effizienz von Leistungserbringern, über die Krankenkassen verfügen können, sind für das Gesundheitswesen wertvoll, weil sie zwar indirekt ermittelt werden, aber sich dafür auf große Zahlen stützen können. Daraus lässt sich jedoch kaum die Schlussfolgerung ziehen, dass die Krankenkassen für ihre Versicherten eine Vorauswahl der wählbaren Ärzte treffen sollten. Stattdessen wäre es nahe liegend, diese Informationen den Versicherten zur Verfügung zu stellen und ihre autonome Entscheidung dadurch zu unterstützen. Dies würde ebenfalls eine Intensivierung des Wettbewerbs zwischen Leistungserbringern bewirken.

Diese Ansätze stellen kein rundes Komplettmodell eines effizienten Krankenversicherungssystems dar, zumal sie den Aspekt der Kostenbegrenzung nicht enthalten. Zu klären, inwieweit dieser durch Wettbewerbsmechanismen zwischen Krankenkassen einzubauen ist, ohne zu sehr mit dem Ziel hoher medizinischer Qualität in Konflikt zu geraten, ist weiterer Forschung vorbehalten. 


\section{Literaturverzeichnis}

Bahrs, O. (2003): Mein Hausarzt hat Zeit für mich - Wunsch und Wirklichkeit, in: G+G Wissenschaft 3. Jg. (2003), Heft 1, S. 17-23.

Baur, R.; Stock, J. (2002): Neue Formen der Krankenversicherung in der Schweiz - zur Evaluation der ersten HMOs in Europa, in: K.-J. Preuß, J. Räbiger, J. H. Sommer (Hrsg.), Managed Care, Evaluation und Performance-Measurement integrierter Versorgungsmodelle. Stuttgart, New York, S. 135-152.

Breyer, F.; Franz, W.; Homburg, S.; Schnabel, R.; Wille, E. (2004): Reform der sozialen Sicherung. Berlin u. a.

Cassel, D. (2005): Ordnungspolitische Reformoptionen im deutschen Gesundheitswesen: Wo liegt Toulon?, in: H. Leipold, D. Wenzel (Hrsg.), Ordnungsökonomik als aktuelle Herausforderung. Schriften zu Ordnungsfragen der Wirtschaft Band 78. Stuttgart, S. 243-261.

Cassel, D.; Ebsen, I.; Greß, S.; Jacobs, K.; Schulze, S.; Wasem, J. (2006): Weiterentwicklung des Vertragswettbewerbs in der gesetzlichen Krankenversicherung, Vorschläge für kurzfristig umsetzbare Reformschritte. Gutachten im Auftrag des AOK-Bundesverbandes, Juli 2006.

Coulter, A.; Cleary, P. D. (2001): Patients' Experiences With Hospital Care In Five Countries, in: Health Affairs Vol. 20 (3), pp. 244-252.

Deutsche Bundesbank (2004): Finanzielle Entwicklung und Perspektiven der gesetzlichen Krankenversicherung, in: Deutsche Bundesbank, Monatsbericht, 56. Jg., Nr. 7, Juli 2004, S. 15-32.

Enthoven, A. C. (1993): The History and Principles of Managed Competition, in: Health Affairs. Supplement September 1993, pp. 24-48.

Europäische Kommission (2007): MISSOC, Gegenseitiges Informationssystem zur sozialen Sicherheit, Soziale Sicherheit in den Mitgliedstaaten der Europäischen Union, im Europäischen Wirtschaftsraum und in der Schweiz, vergleichende Tabellen, im Internet unter: http://ec.europa.eu/employment_social/social_protection/issoc_tables_de.htm, zugegriffen im Dezember 2007.

European Observatory on Health Systems and Policies, Health Systems in Transition Country Profiles, WHO European Centre for Health Policy, Brüssel, im Internet unter: http://www.euro.who.int/observatory/Hits/TopPage, zugegriffen im Dezember 2007.

Greß, S. (2004): Competition in Social Health Insurance: A Three-County Comparison. Diskussionsbeiträge aus dem Fachbereich Wirtschaftswissenschaften der Universität Duisburg-Essen. Campus: Essen, Nr. 135.

Greß, S.; Okma, K.; Hessel, F. (2001): Managed Competition in Health Care in The Netherlands and Germany - Theoretical Foundations, Empirical Findings and Policy Conclusions. Diskussionspapier 04/2001, Lehrstuhl für Allgemeine Be- 
triebswirtschaftslehre und Gesundheitsmanagement der Ernst-Moritz-ArndtUniversität Greifswald.

Grol, R.; Wensing, M.; Mainz, J.; Jung, H. P.; Ferreira, P.; Hearnshaw, H.; Hjordahl, P.; Olesen, F.; Reis, S.; Ribacke, M.; Szecsenyi, J. (2000): Patients in Europe evaluate general practice care: an international comparison, in: British Journal of General Practice 50, pp. 882-887.

Hellinger, F. J. (1998): The Effect of Managed Care on Quality. A Review of Recent Evidence, in: Arch Intern Med (158), pp. 833-841.

Jacobs, K.; Schulze, S. (2004): Wettbewerbsperspektiven integrierter Versorgung in der gesetzlichen Krankenversicherung, in: D. Cassel (Hrsg.), Wettbewerb und Regulierung im Gesundheitswesen. Tagungsband des Ausschusses für Gesundheitsökonomie des Vereins für Socialpolitik. Baden-Baden, S. 89-110.

Klingenberger, D. (2002): Health Maintenance Organizations in der Schweiz - Darstellung und Kritik. IDZ-Information 1/2002.

Miller, R. H.; Luft, H. S. (1997): Does Managed Care Lead To Better Or Worse Quality Of Care?, in: Health Affairs 16 (5), pp. 7-25.

Miller, R. H.; Luft, H. S. (2002): HMO Plan Performance Update: An Analysis Of The Literature, 1997-2001, in: Health Affairs 21 (4), pp. 63-86.

OECD: Gesundheitsdaten 2007. Statistiken und Indikatoren für 30 Länder auf CDROM. Paris.

Perneger, T.; Etter, J.-F.; Schaad, B. (1995): Evaluation der HMO der Universität Genf, Institut für Sozial- und Präventivmedizin (Universität Genf), Genf, im Internet unter: www.gmo.ch/delta/de/hmo\%20genf.htm.

Sachverständigenrat zur Begutachtung der Entwicklung im Gesundheitsweisen (2005): Koordination und Qualität im Gesundheitswesen. Gutachten 2005, Bundestagsdrucksache $15 / 5670$.

Ulrich, V. (2004): Verwaltungskosten der GKV. Janusköpfige Bürokratie oder verordnete Ausgabenfalle?, in: Hessisches Ärzteblatt 3/2004, S. 118-121.

Von Hayek, F. A. (1968): Der Wettbewerb als Entdeckungsverfahren, Kieler Vorträge gehalten an dem Institut für Weltwirtschaft an der Universität Kiel. Kiel.

Wille, E. (1999): Auswirkungen des Wettbewerbs auf die gesetzliche Krankenversicherung, in: E. Wille (Hrsg.), Zur Rolle des Wettbewerbs in der gesetzlichen Krankenversicherung. Baden-Baden, S. 95-156.

Wissenschaftlicher Beirat beim Bundesministerium für Wirtschaft und Technologie (2006): Mehr Wettbewerb im System der Gesetzlichen Krankenversicherung. 